\title{
Badeotitis
}

\section{Rasche Hilfe durch lokale Anwendung von Ohrentropfen}

_ Die akute Otitis externa ist eine schmerzhafte Entzündung des äußeren Ohrs und tritt saisonal gehäuft in der Sommer- und Badezeit auf. Neben

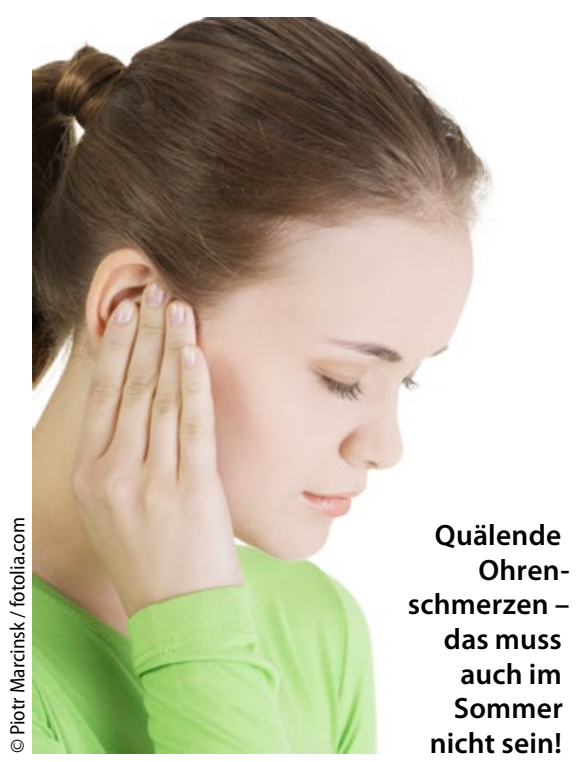

Schwimmern und Tauchern gehören aber auch Träger von Hörgeräten, Allergiker (insbesondere bei Hautekzemen), Diabetiker und Patienten mit chronischeitriger Otitis media zur Risikogruppe der akuten Otitis externa. Therapeutisch empfehlen sich die Beseitigung der Bakterien und abschwellende Maßnahmen zur Schmerzlinderung.

\section{Leitlinien empfehlen lokale antibiotische Therapie}

Die HNO-Leitlinien [Antibiotikatherapie der Infektionen an Kopf und Hals. S2Leitlinie, AWMF-Nr. 017/066, November 2008] empfehlen zur Beseitigung der häufigsten Erreger eine antibiotische Lokaltherapie. Bewährt hat sich dabei der Wirkstoff Ciprofloxacin. Idealerweise sollten die Tropfen den Gehörgang gut benetzen und ausreichend lange haften, unbedenkliche Inhaltsstoffe haben und einen an das Ohrmilieu angepassten sauren $\mathrm{pH}$-Wert.
Mit Panotile ${ }^{\circledast}$ Cipro Ohrentropfen steht ein Präparat mit lipophilen und guten viskösen Eigenschaften, einem ans Ohrmilieu angepasstem $\mathrm{pH}$-Wert von 5,3-6,3 und dem abschwellenden Hilfsstoff Glycerol zur Verfügung. Eine Praxisstudie mit 4.911 Patienten konnte zeigen, dass die Therapie mit Panotile ${ }^{\varpi}$ Cipro bei akuter Otitis externa bereits nach wenigen Tagen eine relevante Schmerzreduktion bei über $90 \%$ der Patienten bewirkte.

Panotile ${ }^{\circledast}$ Cipro kommt dank Einmaldosisbehältnissen ohne potenziell ototoxische Konservierungsstoffe aus. Die hohe Therapiesicherheit spiegelt sich auch in der Tatsache wider, dass es als einziges Präparat auch zur leitliniengerechten lokalen Therapie der chronisch eitrigen Otitis media mit Trommelfelldefekt zugelassen ist.

Red.

- Nach Informationen von Pierre Fabre

\section{HIV-Infektion}

\section{Einmal täglich Raltegravir funktioniert}

- Für Dr. Heribert Knechten, Aachen, bedeutete die Zulassung des Integraseinhibitors Raltegravir (Isentress ${ }^{\circledR}$ ) im Jahr 2007 „einen Meilenstein für die HIV-Patienten und behandelnden Ärzte". Neben der raschen und andauernden Wirksamkeit, der guten Verträglichkeit und dem geringen Interaktionspotenzial mit anderen Arzneimitteln sei eine Integraseinhibitor-basierte HIV-Therapie mittlerweile eine in Leitlinien bevorzugte Therapieoption.

Raltegravir wurde 2007 als erster Integraseinhibitor in Kombination mit weiteren antiretroviralen Medikamenten zugelassen. Mittlerweile erlauben Indikationserweiterungen nicht nur den Einsatz bei erwachsenen HIV-Patienten, sondern auch bei Jugendlichen, Kindern und Säuglingen, die älter als vier Wochen sind. PD Dr. Rika Draenert, Mün- chen, erinnerte daran, dass es neben der 400-mg-Tablette für die zweimal tägliche Standarddosierung auch Kautablette mit $25 \mathrm{mg}$ oder $100 \mathrm{mg}$ und ein Granulat mit Bananen- und Orangengeschmack für die gewichtsadaptierte Dosierung gibt.

Zusätzlich steht nun eine neue Formulierung zur Verfügung, die auch eine einmal tägliche Einnahme erlaubt, berichtete Draenert. Zurzeit wird die Wirksamkeit und Verträglichkeit dieses vereinfachten Therapieschemas in der Phase-III-Studie ONCEMRK mit 750 therapienaiven HIV-Patienten untersucht [www.clinicaltrials.gov; Studiennummer NCTj02131233]. Die Patienten erhalten entweder $1 \times$ täglich $1.200 \mathrm{mg}$ Raltegravir (2 Tabletten à $600 \mathrm{mg}$ ) oder $2 \times$ täglich $400 \mathrm{mg}$ Raltegravir, jeweils in Kombination mit $1 \times$ täglich Tenofovir/
Emtricitabin. Laut einer Pressemeldung des Unternehmens wurden nach 48 Wochen alle Endpunkte der Studie erreicht. Bei der virologischen Wirksamkeit (Anzahl der Patienten mit < 40 HIV-RNAKopien/ml, primärer Endpunkt) war die einmal tägliche Gabe der zweimal täglichen nicht unterlegen. Auch bei den sekundären Endpunkten (immunologische Wirksamkeit und Verträglichkeit) waren beide Formulierungen des Integraseinhibitors vergleichbar.

Die detaillierten Studienergebnisse sollen demnächst vorgestellt und die Anträge auf Marktzulassung in den USA und in Europa noch in diesem Jahr eingereicht werden.

Andrea Warpakowski

- Satellitensymposium "First come, first serve",

16. Münchner AIDS- und Hepatitis-Tage; München, März 2016 (Veranstalter:MSD) 